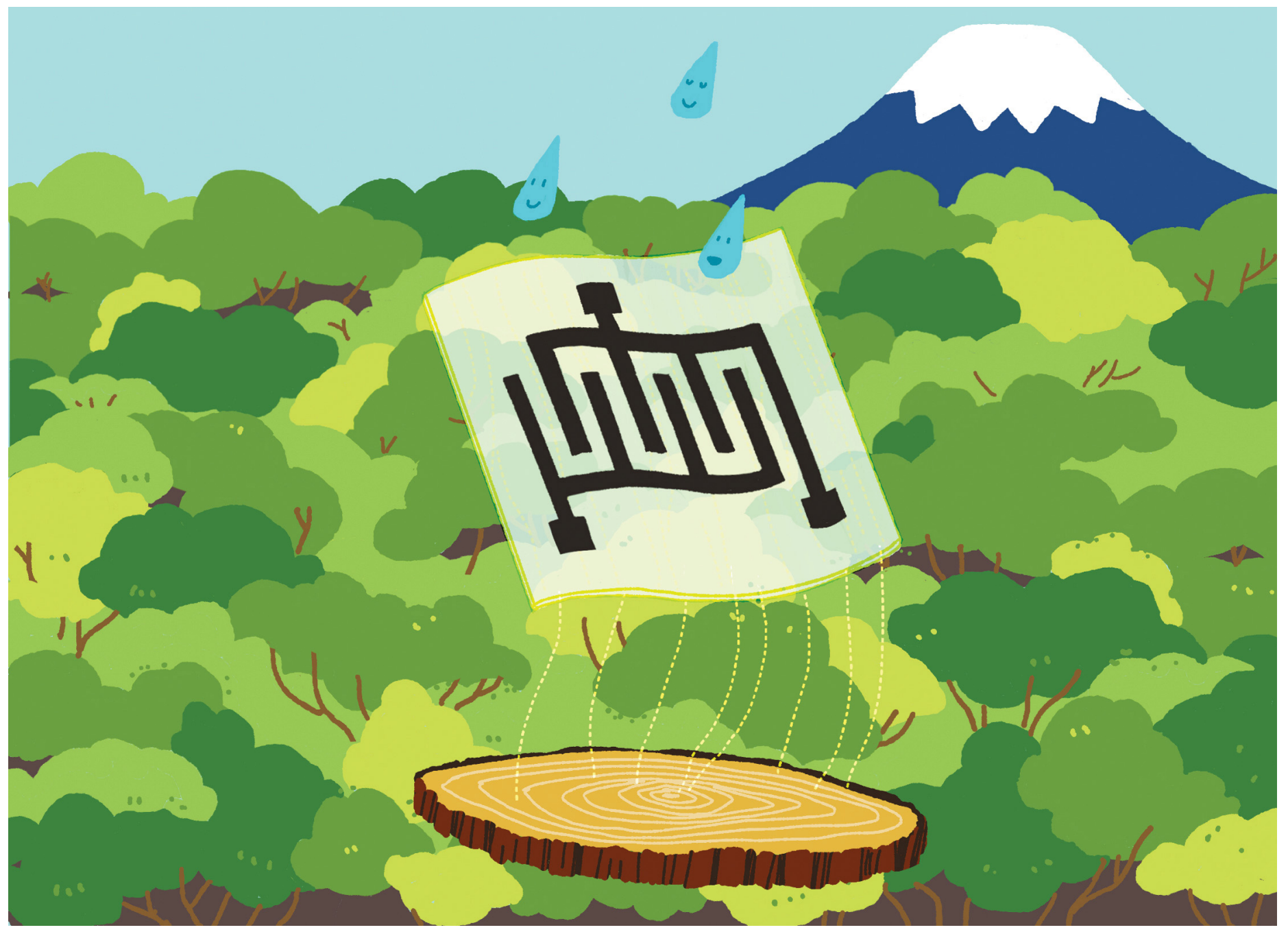

Showcasing research from SANKEN (The Institute of Scientific and Industrial Research), Osaka University, Japan.

All-cellulose-derived humidity sensor prepared via direct laser writing of conductive and moisture-stable electrodes on TEMPO-oxidized cellulose paper

A wood cellulose-derived, abundant, and renewable humidity sensor offers high sensitivity and diverse applicability, paving the way for future sustainable electronics.

\section{As featured in:}

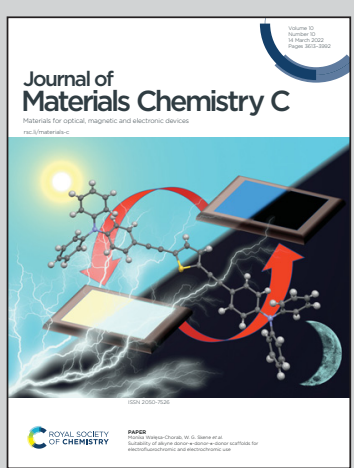

See Hirotaka Koga et al., J. Mater. Chem. C, 2022, 10, 3712. 
Check for updates

Cite this: J. Mater. Chem. C, 2022, 10,3712

Received 4th November 2021, Accepted 28th December 2021 DOI: $10.1039 / \mathrm{d} 1 \mathrm{tc} 05339 f$

rsc.li/materials-c

\title{
All-cellulose-derived humidity sensor prepared via direct laser writing of conductive and moisture-stable electrodes on TEMPO-oxidized cellulose paper $\dagger$
}

\author{
Luting Zhu, (D) Xiang Li, (D) Takaaki Kasuga, (D) Kojiro Uetani, (D) Masaya Nogi and \\ Hirotaka Koga (D) *
}

\begin{abstract}
Humidity sensing plays an important role in diverse applications in the fields of agriculture, healthcare, skincare, and environmental monitoring. With the advent of the "trillion sensor" society, there is a growing demand for humidity sensors that can be made from abundant and renewable resources. Cellulose, the most abundant and renewable bioresource on Earth, acts as a humidity-sensing material because of its hydrophilic nature. However, non-renewable noble metal electrodes are still indispensable because good electrical conductivity and moisture stability are necessary for reliably detecting electrical signals from cellulose-based humidity-sensing materials. Herein, we demonstrate the direct $\mathrm{CO}_{2}$-laser writing of electrodes onto TEMPO-oxidized cellulose paper to realize an all-cellulose-derived humidity sensor. The TEMPO-oxidized cellulose paper with sodium carboxylate groups provides a satisfactory humidity-sensing performance and is converted to conductive and moisture-stable electrodes directly via laser-induced carbonization. The resulting all-cellulose-derived humidity sensor demonstrates high sensitivity and linearity over a wide range of relative humidity (11-98\%), thereby providing broad applicability. The sensor can be used for the positional localization of moist objects and for monitoring plant transpiration and human-body sweating. These results open the door for renewable and sustainable humidity sensors.
\end{abstract}

\section{Introduction}

With the advent of the "trillion sensor" era, the global use of sensing devices has rapidly increased. Humidity sensors are a major attraction and have diverse applications such as in industrial instrumentation and automation, agriculture, healthcare, skincare, and climate monitoring. ${ }^{1,2}$ The market size of humidity sensors is estimated to grow from USD 4.0 billion in 2019 to USD 11.85 billion by $2027 .{ }^{3}$ The production of humidity sensors is expected to increase concomitantly; therefore, there is a need for the development of new sensors using abundant, ubiquitous, and even renewable materials.

Humidity sensors generally comprise a humidity-sensing material and electrodes. ${ }^{4}$ Humidity-sensing materials, such as ceramics $\left(\mathrm{Al}_{2} \mathrm{O}_{3}, \mathrm{SiO}_{2}\right.$, etc. $)$, semiconductors $\left(\mathrm{TiO}_{2}, \mathrm{ZnO}, \mathrm{SnO}_{2}\right.$,

SANKEN (The Institute of Scientific and Industrial Research), Osaka University, 8-1 Mihogaoka, Ibaraki, Osaka 567-0047, Japan.

E-mail: hkoga@eco.sanken.osaka-u.ac.jp; Fax: +81-6-6879-8444; Tel: +81-6-6879-8442

$\dagger$ Electronic supplementary information (ESI) available. See DOI: 10.1039/ d1tc05339f
$\mathrm{In}_{2} \mathrm{O}_{3}$, perovskites, etc.), and polymers, ${ }^{4,5}$ exhibit a change in their electrical signal in response to the adsorption and desorption of water vapor. ${ }^{6}$ Electrodes, particularly interdigitated electrodes, detect the electrical signal from the humiditysensing material. Because electrodes require sufficient electrical conductivity and stability against moisture, they are commonly made from noble metals, such as platinum, ${ }^{7}$ gold, ${ }^{8,9}$ and silver. ${ }^{10,11}$ However, to meet the demand of the "trillion sensor" era, humidity sensors must be composed of abundant and renewable resources.

Cellulose is the most abundant and renewable bioresource in the world. Moreover, it is promising for use in humidity sensors. ${ }^{12}$ As the raw material for paper, cellulose can be applied as a substrate in various electronic devices, including humidity sensors. ${ }^{13-16}$ In addition, the high density of hydrophilic groups in cellulose molecules makes them sensitive to water vapor, thus providing the ability to use cellulose as a humidity-sensing material. ${ }^{11,17-20}$ Various electrodes have been fabricated on cellulosic materials using different methods, ${ }^{21}$ such as printing, ${ }^{11}$ pasting, ${ }^{20}$ painting, ${ }^{22,23}$ and spraying, ${ }^{24}$ for flexible and wearable humidity sensing. For example, a flexible humidity sensor comprising a 2,2,6,6-tetramethylpiperidine-1-oxyl 
radical (TEMPO)-oxidized cellulose nanofiber as the sensing material and gold as the electrodes has been reported. ${ }^{17}$ Degradable humidity sensors have also been developed using cellulose nanofiber paper as the sensing material and silver as the electrodes. ${ }^{11}$ However, the use of non-renewable electrodes is still necessary to meet many requirements, such as having a sufficiently higher electrical conductivity than humiditysensing materials and stability against moisture, making it difficult to realize humidity sensors made from fully renewable materials.

Herein, we demonstrate the all-cellulose-derived renewable humidity sensor. TEMPO-oxidized cellulose paper is used as a humidity-sensing material, onto which electrodes are fabricated in situ via $\mathrm{CO}_{2}$-laser-induced carbonization of the TEMPO-oxidized cellulose. The laser-induced carbonization of TEMPO-oxidized cellulose with sodium carboxylate (COONa) groups produces adequately conductive and even moisturestable electrodes, while the TEMPO-oxidized cellulose paper with COONa groups provides suitable humidity-sensing properties to realize an all-cellulose-derived humidity sensor. The resulting humidity sensor exhibits high sensitivity and linearity, and a large dynamic range. It also has diverse applicability, such as in the detection of moist objects and the monitoring of biological activities.

\section{Results and discussion}

In situ formation of conductive and moisture-stable electrodes onto TEMPO-oxidized cellulose paper via $\mathrm{CO}_{2}$-laser irradiation

All-cellulose-derived humidity sensors were fabricated following the workflow shown in Fig. 1a. We first prepared TEMPOoxidized cellulose paper by oxidizing softwood pulp fibers in a TEMPO-mediated system, ${ }^{25,26}$ after which they were suspended in water with gentle stirring. The suspension was then filtered, transferred to a glass plate, and oven-dried to form TEMPOoxidized cellulose paper with a COONa group content of approximately $1.8 \mathrm{mmol} \mathrm{g}^{-1}$ (Fig. S1, ESI $\dagger$ ). Thereafter, we directly formed $\mathrm{CO}_{2}$-laser-induced electrodes on the TEMPOoxidized cellulose paper via irradiation using a continuous $\mathrm{CO}_{2}$ laser under atmospheric conditions, according to a designed irradiation pattern (Fig. S2, ESI $\dagger$ ). The laser-irradiated area turned black, providing submillimeter-scale line patterning of an interdigitated structure with a similar size to the designed irradiation pattern (Fig. 1b). The electrical conductivity of the laser-irradiated area changed with the laser power at a fixed scan rate of $10 \mathrm{~cm} \mathrm{~s}^{-1}$ (Fig. 1c). While the original TEMPOoxidized cellulose paper had a high surface resistance of approximately $10^{9} \Omega \mathrm{sq}^{-1}$, the laser-irradiated area had a surface resistance of $10^{8}-10^{2} \Omega \mathrm{sq}^{-1}$, depending on the laser power.

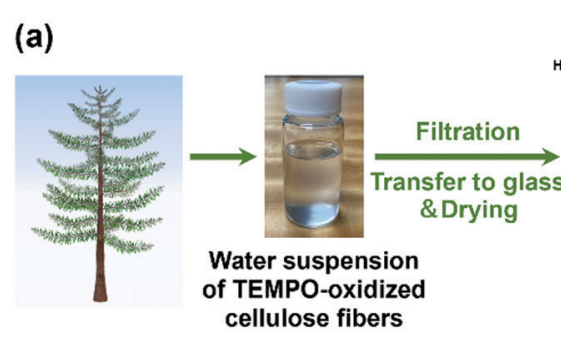

(b)

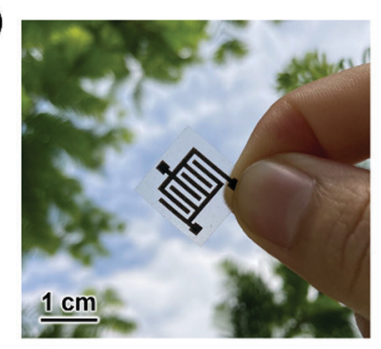

(c)

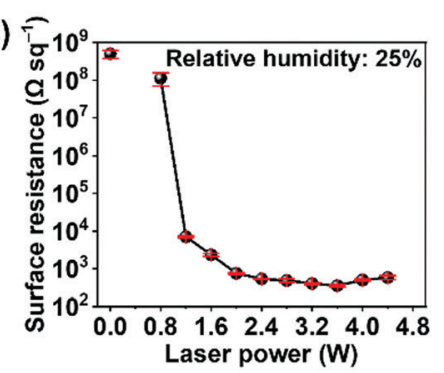

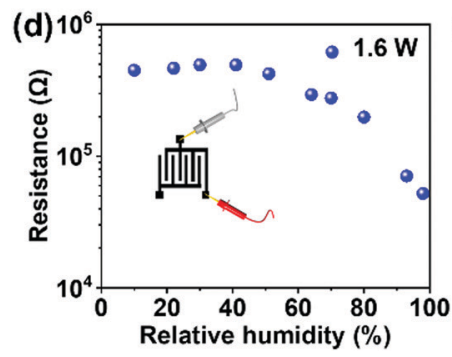
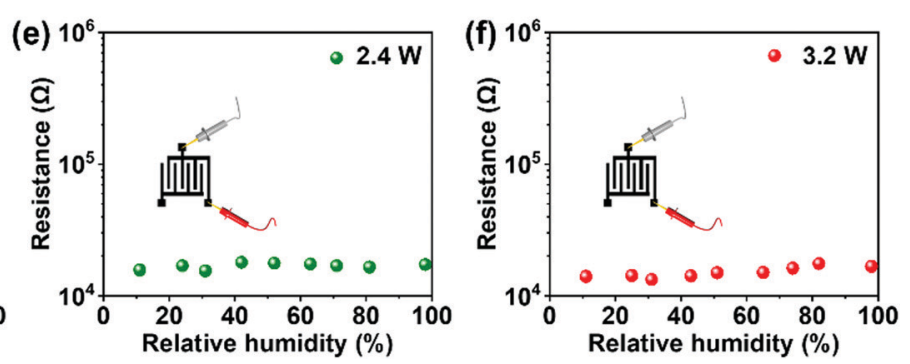

Fig. $1 \mathrm{CO}_{2}$-laser-induced direct formation of electrodes on TEMPO-oxidized cellulose paper. (a) Schematic of the preparation and $\mathrm{CO}_{2}$-laser irradiation of TEMPO-oxidized cellulose paper, (b) optical image of the resulting paper, (c) surface resistance as a function of laser power used for laser irradiation, $(d-f)$ relationship between relative humidity and electrical resistance of laser-induced electrodes prepared at laser powers of (d) 1.6, (e) 2.4, and (f) 3.2 W. Laser scan speed: $10 \mathrm{~cm} \mathrm{~s}^{-1}$. The resistance was measured at room temperature. 
This facilitated the formation of conductive electrodes on the TEMPO-oxidized cellulose paper. When the laser power exceeded 4.0 $\mathrm{W}$, there was a slight increase in the surface resistance, suggesting that excess laser energy is undesirable for preparing conductive electrodes (see also Fig. S3, ESI $\dagger$ ).

We further evaluated the moisture stability of the electrodes, which is a critical property for humidity-sensing electrodes. The electrode prepared using a laser power of $1.6 \mathrm{~W}$ retained its resistance up to a relative humidity of $40 \%$, after which its resistance decreased with a further increase in relative humidity. This indicates a lack of moisture stability (Fig. 1d). By contrast, the electrodes prepared at 2.4 and $3.2 \mathrm{~W}$ laser power exhibited stable resistance regardless of the relative humidity (Fig. 1e and f). Thus, conductive and moisturestable electrodes were successfully patterned on the TEMPOoxidized cellulose paper via $\mathrm{CO}_{2}$-laser irradiation and power tuning.

To explain the $\mathrm{CO}_{2}$-laser-induced formation of the electrodes, we analyzed the changes in the surface and molecular structures of the TEMPO-oxidized cellulose paper upon $\mathrm{CO}_{2}$ laser irradiation using field-emission scanning electron microscopy (FE-SEM), energy dispersive X-ray spectroscopy (EDX), Fourier-transform infrared (FT-IR) spectroscopy and Raman spectroscopy (Fig. 2). As shown in Fig. 2a-d, the surface structure became porous after $\mathrm{CO}_{2}$-laser irradiation. In addition, the oxygen/carbon $(\mathrm{O} / \mathrm{C})$ atomic ratio decreased with increasing laser-irradiation power (Fig. 2e), indicating the progress of carbonization by the removal of oxygen. FT-IR analysis showed that the original molecular structure disappeared after $\mathrm{CO}_{2}$-laser irradiation (Fig. 2f). At a laser power of $1.6 \mathrm{~W}$, a $\mathrm{C}=\mathrm{C}$ peak appeared, while $\mathrm{CO}_{3}{ }^{2-}$ peaks were observed due to the presence of $\mathrm{Na}_{2} \mathrm{CO}_{3} \cdot{ }^{27,28}$ At higher laser powers $(2.4$ and $3.2 \mathrm{~W})$, no $\mathrm{CO}_{3}{ }^{2-}$ peaks were observed. In the Raman spectra, the intensities of the $\mathrm{G}$ and $\mathrm{D}$ bands, which are associated with ordered and disordered graphitic carbon structures, respectively, ${ }^{29}$ increased with increasing laser power (Fig. 2g). After irradiation at a laser power of $3.2 \mathrm{~W}$, the $2 \mathrm{D}$ band appeared, which is associated with the stacking structure of graphitic carbon; ${ }^{30,31}$ therefore, this indicates the formation of a more graphitized carbon structure. These results indicate that the $\mathrm{CO}_{2}$-laser-induced carbonization of the TEMPO-oxidized cellulose progressed with increasing laser power, leading to the formation of more conductive electrodes. The low moisture stability of the $\mathrm{CO}_{2}$-laser-induced electrodes produced at $1.6 \mathrm{~W}$ (Fig. 1d) could be attributed to the presence of water-soluble $\mathrm{Na}_{2} \mathrm{CO}_{3}$; their resistance decreases at high relative humidity possibly by the ionic conduction of the dissolved $\mathrm{Na}^{+}$. The $\mathrm{CO}_{2}$-laser-induced conductive electrodes produced at 2.4 and 3.2 W laser power were electrically stable against moisture (Fig. 1e and $\mathrm{f}$ ), although they adsorbed water molecules on their surfaces (Fig. S4, ESI $\dagger$ ). Thus, their moisture stability would be provided by maintaining the original conductive pathways derived from a more graphitized carbon structure within the electrodes, even after water adsorption.

The $\mathrm{CO}_{2}$-laser-induced carbonization of polymeric materials can be attributed to the generation of thermal energy due to the photothermal effect derived from their lattice vibrations. ${ }^{30}$ When subjected to $\mathrm{CO}_{2}$-laser irradiation, the polymeric material reaches a high temperature, which causes chemical bonds such as $\mathrm{C}-\mathrm{O}$ and $\mathrm{C}=\mathrm{O}$ within the polymer to break and rearrange to form a graphitic structure. ${ }^{30}$ The use of $\mathrm{CO}_{2}$-laserinduced carbonization to achieve conductive patterning was first reported for polyimide. ${ }^{30}$ In this study, the $\mathrm{CO}_{2}$-laser-induced

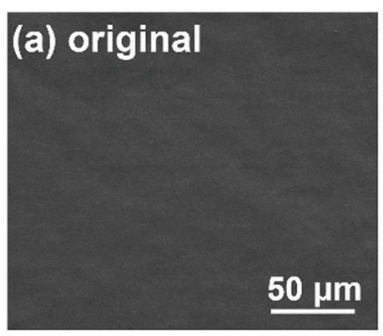

(e) 0

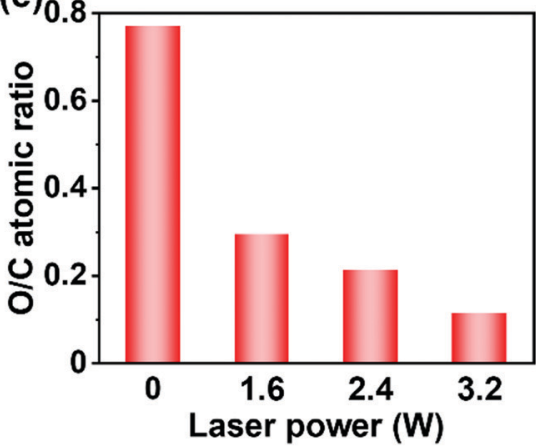

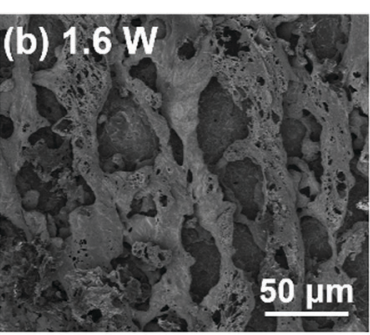
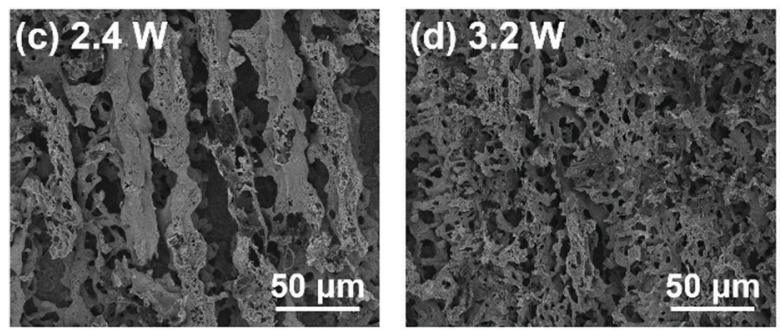

(g)
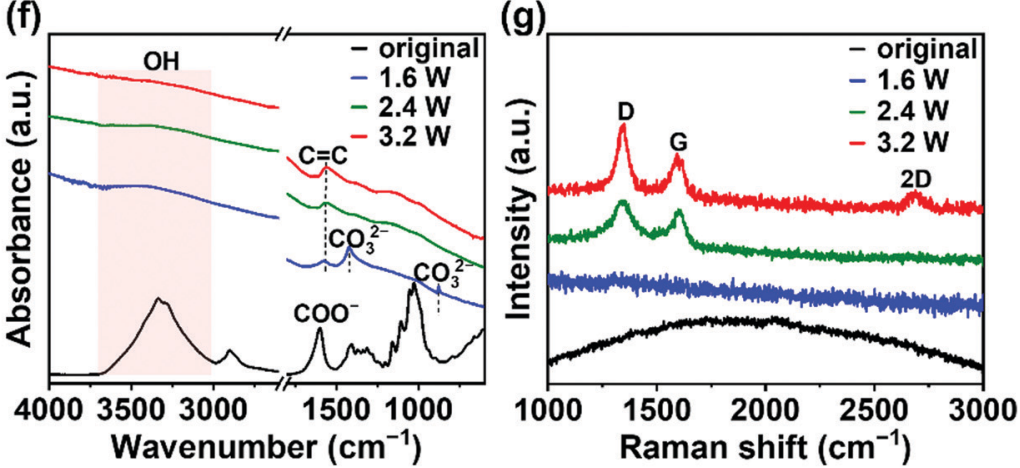

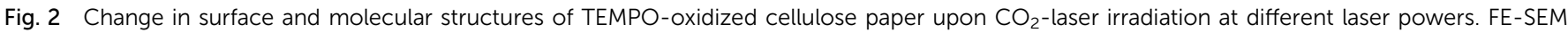

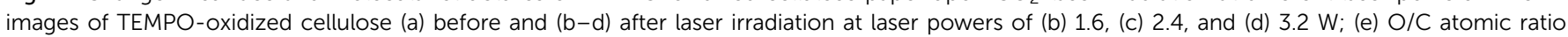
estimated by EDX, (f) FT-IR spectra, and (g) Raman spectra. 
formation of conductive electrodes was successfully performed on TEMPO-oxidized cellulose with COONa groups. However, this was not possible on the original cellulose or even TEMPO-oxidized cellulose with $\mathrm{COOH}$ groups, owing to ablation by the $\mathrm{CO}_{2}$-laser irradiation (Fig. S5, ESI $\dagger$ ). Thermogravimetric analysis showed that TEMPO-oxidized cellulose with COONa groups had a considerably higher yield of carbonaceous materials after hightemperature treatment than the original cellulose and TEMPOoxidized cellulose with $\mathrm{COOH}$ groups (Fig. S6, ESI $\dagger$ ). Thus, COONa groups suppress the ablation of TEMPO-oxidized cellulose under $\mathrm{CO}_{2}$-laser irradiation, thereby allowing it to be carbonized to form conductive electrodes. While there have been several reports on the $\mathrm{CO}_{2}$-laser carbonization of TEMPOoxidized cellulose with COONa groups, ${ }^{32}$ our results highlight the formation of conductive and even moisture-stable electrodes for humidity-sensing applications.

\section{All-cellulose-derived humidity sensor}

We evaluated the humidity-sensing performance of the allcellulose-derived sensing device comprising TEMPO-oxidized cellulose paper as the sensing material and $\mathrm{CO}_{2}$-laser-induced interdigitated electrodes (Fig. 3a, see also Fig. S2, ESI $\dagger$ ). The interdigitated electrodes afforded the stable detection of electrical signals from TEMPO-oxidized cellulose paper (see Fig. S7, ESI $\dagger$ for details). As shown in Fig. $3 \mathrm{~b}$ and Fig. S8 (ESI $\dagger$ ), the device showed a clear response to water vapor at room temperature; the electrical resistance decreased quickly upon exposure to water vapor from a humidifier and recovered to the original resistance when the humidifier was removed from the atmosphere. The recovery time clearly varied depending on the irradiating power used to form the laser-induced electrodes. The laser-induced electrodes produced at $1.6 \mathrm{~W}$ exhibited a long recovery time (approximately $470 \mathrm{~s}$ ), suggesting that the resistance change of the electrodes themselves against water vapor delayed the recovery. By contrast, the laser-induced electrodes produced at 2.4 and $3.2 \mathrm{~W}$ exhibited a considerably shorter recovery time (approximately 120 and $90 \mathrm{~s}$, respectively). These results indicate that conductive and moisture-stable electrodes can provide fast recovery for repeated sensing in a short period of time.

As depicted in Fig. 3c-e, the sensing devices presented a high log linearity of electrical resistance with good repeatability and reproducibility in a dynamic range of relative humidity, regardless of the power used to produce the laser-induced electrodes. Therefore, the devices acted as resistive-type humidity sensors. The humidity-sensing mechanism is due to the ionic conduction of $\mathrm{Na}^{+}$from the COONa groups within the TEMPOoxidized cellulose paper (Fig. 3a). More specifically, higher humidity conditions lead to the increased adsorption of water molecules on the hydrophilic cellulose molecules, ${ }^{33}$ which allows the dissociation of COONa groups and the formation of more mobile pathways for dissociated $\mathrm{Na}^{+}$, leading to decreased resistance. The laser-induced interdigitated electrodes decrease the detected resistance values of the sensing area (TEMPO-oxidized cellulose) to ensure accurate humidity sensing (Fig. S7, ESI $\dagger$ ). As such, the humidity sensor with laserinduced interdigitated electrodes formed at $3.2 \mathrm{~W}$ offered high linearity over a dynamic range of relative humidity (11-98\%). The performance was comparable to that of a TEMPO-oxidized cellulose device with sputtered platinum electrodes (Fig. S9, ESI $\dagger$ ), indicating that laser-induced electrodes act as a suitable substitute for conventional precious-metal electrodes. The sensitivity and response/recovery time of the all-cellulosederived humidity sensor, which were evaluated under two different (a)

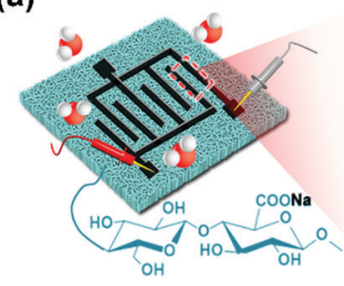

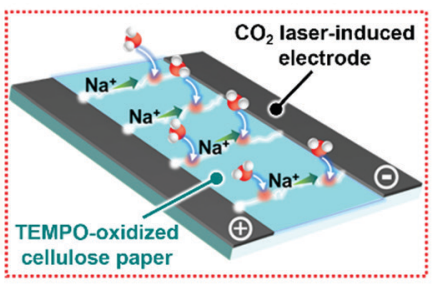

(b)

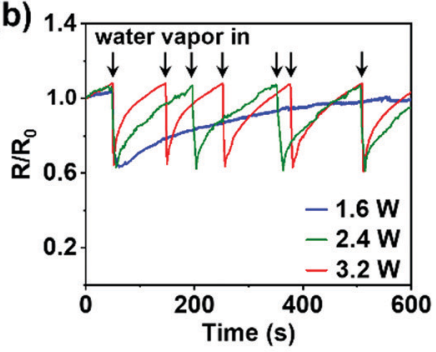

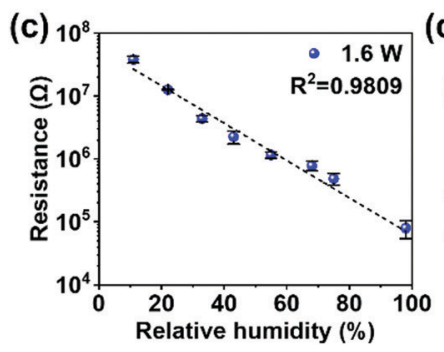
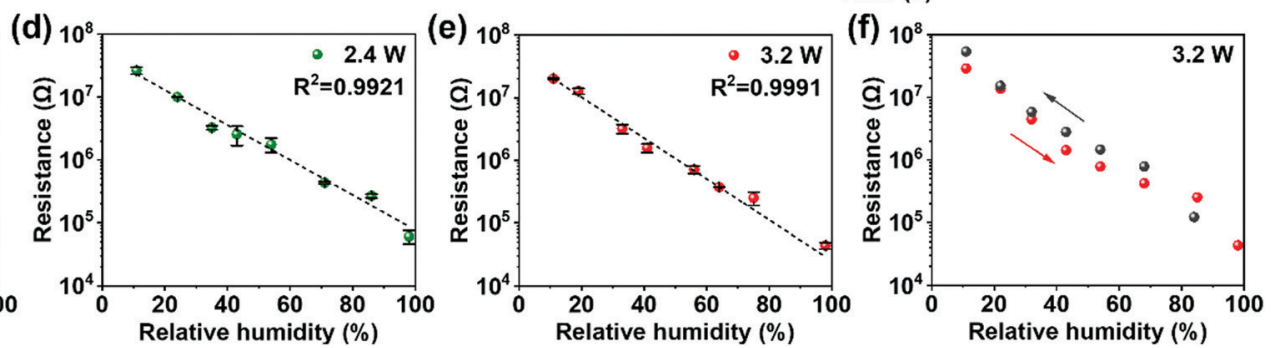

Fig. 3 Sensing performance of all-cellulose-derived humidity sensors comprising TEMPO-oxidized cellulose and $\mathrm{CO}_{2}$-laser-induced electrodes formed at different $\mathrm{CO}_{2}$-laser-irradiation powers. (a) Schematic illustration and putative humidity-sensing mechanism of the all-cellulose-derived humidity sensor, (b) relative resistance versus time of sensors exposed to water vapor from a humidifier (relative humidity: $50 \%$ ), (c-e) resistance versus relative humidity of sensors formed using laser-irradiation powers of (c) 1.6, (d) 2.4, and (e) $3.2 \mathrm{~W}$, and (f) hysteresis property of the all-cellulose-derived humidity sensor formed using a laser-irradiation power of $3.2 \mathrm{~W}$. All performance tests were conducted at room temperature. 
relative humidity conditions (30 and $75 \%$ ), were $1.19 \times 10^{5}$ and $60 / 495$ s, respectively (Fig. S10, ESI $\dagger$ ). The relatively long time for recovery would be due to the hydrophilic nature of cellulose that required more time to desorb the water molecules. Nevertheless, the all-cellulose-derived humidity sensor exhibited high sensitivity, as well as adequate response and recovery times, as compared to state-of-the-art cellulose-based humidity sensors that use non-renewable electrodes (Table S1, ESI $\dagger$ ). Moreover, the all-cellulose-derived humidity sensor showed real-time response and recovery upon the change in humidity (Fig. S11, ESI $\dagger$ ), mild hysteresis (Fig. 3f), and stability that is sufficient to maintain its sensing performance after 100 bending cycles (Fig. S12, ESI $\dagger$ ) as well as storing for at least 3 months (Fig. S13, $\mathrm{ESI} \dagger)$. The $\mathrm{CO}_{2}$-laser-irradiation process is extremely attractive for industrial use because it can be entirely performed in ambient air without any reagents. ${ }^{30}$ Thus, a high-performance, renewable, and facile-to-fabricate all-cellulose-derived humidity sensor was successfully demonstrated.

\section{Wide applicability of all-cellulose-derived humidity sensors}

Humidity sensors have become ubiquitous tools for several emerging applications. ${ }^{2}$ Here, we demonstrate the diverse applicability of the proposed all-cellulose-derived humidity sensor. The $\mathrm{CO}_{2}$-laser-irradiation process is a facile method for forming patterned electrodes on TEMPO-oxidized cellulose paper, and can also be used to cut the paper into various shapes, allowing for versatile configurations of humidity sensors (Fig. 4a, see also Fig. S14, ESI $\dagger$ ). By taking advantage of the laser-irradiation process, we fabricated a square humiditysensor array consisting of nine sensor elements (Fig. 4b). When a finger approached the sensor array, the electrical resistance of the sensor elements located near the finger decreased to approximately $70 \%$ owing to the humidity of the skin. As such, the sensor array detected the approaching finger via a change in resistance. This indicates that the sensor array can be applied for positional localization of moist objects.

Owing to the paper-specific advantages of the sensor, such as good handleability, flexibility, and being lightweight, the all-cellulose-derived humidity sensor was applied for the synchronous detection of biological activities. As shown in Fig. 4c, a humidity sensor ring was worn on a finger, which responded to human exhalation-derived water vapor. The humidity sensor could detect the different extents of exhalation (nasal breathing, soft and deep mouth breathing) and could monitor real-time
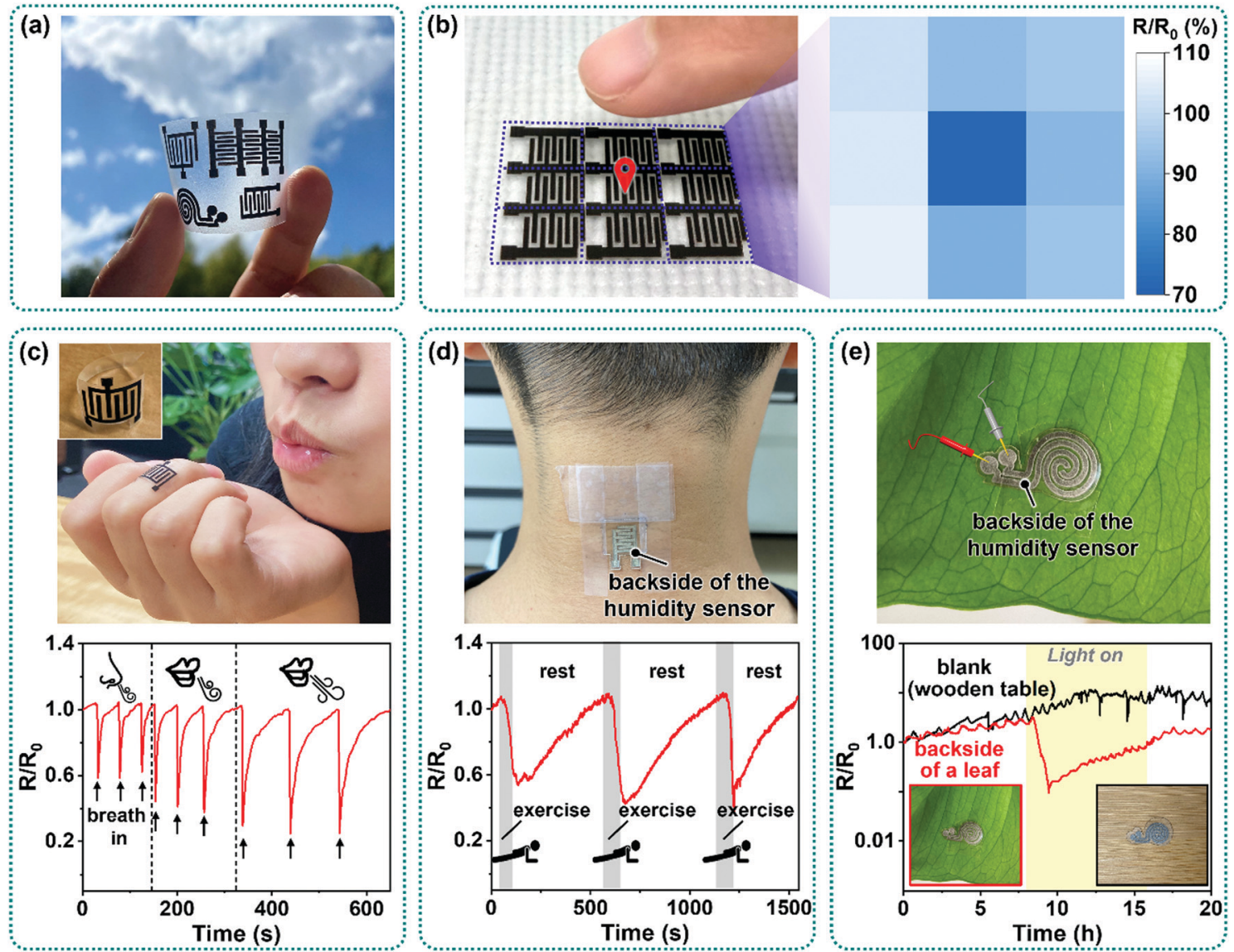

Fig. 4 Applicability of the all-cellulose-derived humidity sensor. (a) Optical image of all-cellulose-derived humidity sensors with various patterned electrodes, (b) positional localization of an approaching finger using a $3 \times 3$ sensor array, (c) sensor response for different extents of human exhalation (breathing from the nose and mouth (soft and hard)), (d) monitoring of human-body sweating process during plank exercise and rest periods, and (e) monitoring of the transpiration process of a Monstera leaf in comparison with monitoring of a wooden table surface. In all cases, the electrodes were prepared at a laser power of $3.2 \mathrm{~W}$. All demonstrations were conducted at room temperature. 
nasal breathing (Fig. S15, ESI $\dagger$ ), suggesting the potential applicability for breathing-behavior analysis related to disease monitoring. ${ }^{34}$ The humidity sensor was also used for monitoring the sweating process from the human body (Fig. 4d). When the sensor was attached to the back of the neck, clear sensor responses were reproducibly observed upon perspiration during repeated plank exercises and rest periods, providing the potential for accurate recovery of the hydration status in daily exercise. ${ }^{35}$

Finally, we applied the humidity sensor for monitoring plant activity. Plant leaves release water vapor by a process known as transpiration, which is enhanced under light illumination. ${ }^{36,37}$ When attached to the reverse side of a Monstera leaf, the relative resistance of the humidity sensor decreased upon visible-light illumination, while no such response was observed when the sensor was applied to a wooden table (Fig. 4e, see also Fig. S16, ESI $\dagger$ ). This indicates that the proposed sensor can monitor plant transpiration. Thus, the all-cellulose-derived humidity sensor meets the requirements of ubiquitous sensing as well as abundance and renewability for the "trillion sensor" era.

\section{Conclusions}

We successfully fabricated an all-cellulose-derived humidity sensor consisting of a TEMPO-oxidized cellulose paper sensor and $\mathrm{CO}_{2}$-laser-induced electrodes. In addition to providing humidity-sensing performance, the TEMPO-oxidized cellulose with COONa groups facilitated the laser-induced formation of conductive and moisture-stable electrodes that provided reliable humidity sensing with adequate recovery for repeated sensing in a short timeframe. The laser process allows fine patterning of the electrodes, and thus the versatile configuration of humiditysensor elements. Combined with the paper-specific properties of flexibility and being lightweight, the all-cellulose-derived humidity sensor demonstrated diverse applicability, including the positional localization of moist matter and the wearable sensing of biological activity. Because cellulose is the most ubiquitous and abundant bioresource on Earth, these results are expected to pave the way for renewable and sustainable humidity sensors in the future. This approach can be also extended to fabricate various electronic devices such as supercapacitors towards all-cellulosederived electronics.

\section{Experimental}

\section{Materials}

Never-dried softwood bleached kraft pulp was kindly provided by Ehime University, Japan. TEMPO was purchased from Tokyo Chemical Industry Co., Ltd (Tokyo, Japan). Sodium bromide $(\mathrm{NaBr})$, sodium hypochlorite ( $\mathrm{NaClO}$ ) solution, and other chemicals were purchased from Nacalai Tesque, Inc. (Kyoto, Japan).

\section{Preparation of TEMPO-oxidized cellulose fibers}

TEMPO-mediated oxidation of softwood cellulose pulp was conducted according to a published method. ${ }^{26}$ Briefly, the cellulose pulp $(20 \mathrm{~g})$ was suspended in distilled water $(2 \mathrm{~L})$, followed by mixing with TEMPO (0.32 g) and $\mathrm{NaBr}(2 \mathrm{~g})$. TEMPO-mediated oxidation was initiated by adding NaClO (15 mmol per gram of cellulose) to the suspension. The $\mathrm{pH}$ of the suspension was maintained at 10 by adding $\mathrm{NaOH}(0.5 \mathrm{M})$ until no $\mathrm{NaOH}$ consumption was observed. Oxidation was quenched by adding ethanol (approximately $100 \mathrm{~mL}$ ). The resulting TEMPO-oxidized cellulose fibers with COONa groups were thoroughly washed with distilled water using vacuum filtration, and stored at $4{ }^{\circ} \mathrm{C}$ without drying.

To prepare TEMPO-oxidized cellulose fibers with $\mathrm{COOH}$ groups, an aqueous suspension of TEMPO-oxidized cellulose fibers with COONa groups was mixed with $\mathrm{HCl}$ aqueous solution to adjust the $\mathrm{pH}$ of the suspension to below 2. The resultant suspension was magnetically stirred for $3 \mathrm{~h}$ and then centrifuged at $9000 \mathrm{rpm}$ for $10 \mathrm{~min}$. The sediment was washed with distilled water and centrifuged. The washing treatment was repeated thrice. Finally, the sediment was dispersed in distilled water to prepare an aqueous suspension of TEMPOoxidized cellulose fibers with $\mathrm{COOH}$ groups.

\section{Preparation of TEMPO-oxidized cellulose paper}

An aqueous suspension of TEMPO-oxidized cellulose fibers $(0.2 \mathrm{wt} \%, 200 \mathrm{~mL})$ was dewatered via vacuum filtration on a membrane filter (H020A090C, hydrophilic polytetrafluoroethylene membrane, $0.2 \mu \mathrm{m}$ pore diameter, Advantec Toyo Kaisha, Ltd, Tokyo, Japan). The obtained wet sheet was peeled from the membrane filter, transferred to a glass plate, and dried using an environmental chamber (SH-642, ESPEC Corp., Osaka, Japan) at a temperature of $20{ }^{\circ} \mathrm{C}$ and relative humidity of $60 \%$.

\section{Preparation of $\mathrm{CO}_{2}$-laser-induced electrodes on TEMPO- oxidized cellulose paper}

TEMPO-oxidized cellulose paper on a glass plate was irradiated using a continuous-wave $\mathrm{CO}_{2}$ laser with a wavelength of $10.6 \mu \mathrm{m}$ (HAJIME CL1 PLUS, Oh-Laser Co., Ltd, Saitama, Japan) under atmospheric conditions to form the laser-induced electrodes. The spot size of the laser beam was $80 \mu \mathrm{m}$. Various electrode patterns were fabricated using a computer-controlled laser program to control the laser power, scan rate, and scan pattern.

\section{Preparation of Pt electrodes on TEMPO-oxidized cellulose paper}

For comparison with the $\mathrm{CO}_{2}$-laser-induced electrodes, Pt electrodes were fabricated on the TEMPO-oxidized cellulose paper by ion sputtering $(20 \mathrm{~mA}, 240 \mathrm{~s}$; E-1045, Hitachi High-Tech Corp., Tokyo, Japan) three times using a mask. The mask was fabricated by $\mathrm{CO}_{2}$-laser cutting of an acrylic sheet (CLAREX, Nitto Jushi Kogyo Co., Ltd, Tokyo, Japan).

\section{Evaluation of humidity-sensing performance}

The humidity-sensing performance was evaluated at room temperature (around $25{ }^{\circ} \mathrm{C}$ ) using an electrochemical workstation (ModuLab XM, Solartron Analytical-AMETEK Advanced Measurement Technology Inc., Berkshire, UK). Time-current curves of the humidity sensors were measured under a voltage 
of $1 \mathrm{~V}$. The humidity sensors were placed in airtight boxes at different relative humidity values. The relative humidity conditions were dynamically controlled using different saturated salt solutions (LiCl, $\mathrm{CH}_{3} \mathrm{COOK}, \mathrm{MgCl}_{2}, \mathrm{~K}_{2} \mathrm{CO}_{3}, \mathrm{Mg}\left(\mathrm{NO}_{3}\right)_{2}, \mathrm{CuCl}_{2}$, $\mathrm{NaCl}, \mathrm{KCl}$, and $\mathrm{K}_{2} \mathrm{SO}_{4}$ for $11 \%, 22 \%, 32 \%, 43 \%, 54 \%, 68 \%$, $75 \%, 85 \%$, and $98 \%$ relative humidity, respectively), and calibrated using a digital temperature and humidity meter (TR-72 wb, T\&D Corporation, Nagano, Japan). Instantaneous exposure of the humidity sensor to water vapor was performed using a humidifier (JANRSTIC Ultrasonic Aroma Humidifier, Dongguan, China). The real-time response and recovery curves in the relative humidity range of $25-68 \%$ were measured by putting the humidity sensor in a bench-top-type temperature and humidity chamber (SH-642, Espec Corp., Osaka, Japan). The positional localization of a finger was determined by measuring the resistance of each humidity sensing part with a digital multimeter (PC 7000, Sanwa Electric Instrument Co., Ltd, Tokyo, Japan). The response and recovery times were measured under two different humidity conditions (30 and 75\%) controlled using different saturated salt solutions. The response and recovery times were defined as the time required to reach $90 \%$ of the change in sensor resistance. ${ }^{1,38}$ The sensitivity $(S)$ was calculated using the following equation: ${ }^{17,39}$

$$
S=\Delta R / \Delta \mathrm{RH}
$$

where $\Delta R$ is the change in the sensor resistance and $\Delta \mathrm{RH}$ is the change in the relative humidity level.

\section{Analyses}

Surface observations were performed using FE-SEM at an accelerating voltage of $2 \mathrm{kV}$ (SU-8020, Hitachi High-Tech Corp., Tokyo, Japan). Platinum sputtering was performed before the FE-SEM observation. EDX analysis was coupled with FE-SEM at an accelerating voltage of $15 \mathrm{kV}$ (EDAX, AMETEK. Inc., New Jersey, USA). The surface resistance was measured using a resistivity meter with a ring-type probe (Hiresta-UX, MCPHT800, Mitsubishi Chemical Analytech Co., Ltd, Tokyo, Japan) and a four-probe resistivity meter (Loresta-GP, MCP-T610, Mitsubishi Chemical Analytech Co., Ltd, Tokyo, Japan). FT-IR/ attenuated total reflection spectra were obtained using a KJP05120S instrument (PerkinElmer Japan Co. Ltd, Kanagawa, Japan). Raman spectra were recorded at a laser wavelength of 532 nm (RAMAN touch VIS-NIR-OUN, Nanophoton Corp., Osaka, Japan). Thermogravimetric analyses were conducted in air $\left(200 \mathrm{~mL} \min ^{-1}\right)$ at a heating rate of $10{ }^{\circ} \mathrm{C} \min ^{-1}$ (TGA Q50N2, TA Instruments, New Castle, USA).

\section{Author contributions}

Luting Zhu: data curation, funding acquisition, investigation, methodology, resources, validation, visualization, and writing original draft; Xiang Li: investigation, and visualization; Takaaki Kasuga: methodology, resources, and writing - review and editing; Kojiro Uetani: resources, and writing - review and editing; Masaya Nogi: resources, supervision, and writing - review and editing; Hirotaka Koga: conceptualization, funding acquisition, methodology, project administration, resources, supervision, visualization, and writing - original draft as well as review and editing.

\section{Conflicts of interest}

There are no conflicts to declare.

\section{Acknowledgements}

This work was partially supported by a Grant-in-Aid for JSPS fellow (JP20J11624 to L. Z.); the "Nanotechnology Platform Project (Nanotechnology Open Facilities in Osaka University)" from the Ministry of Education, Culture, Sports, Science and Technology, Japan (No. JPMXP09S21OS0029 to H. K.); the Japan Prize Heisei Memorial Research Grant Program (H. K.); and JST FOREST Program (JPMJFR2003 to H. K.).

\section{References}

1 Y. Wang, L. Zhang, J. Zhou and A. Lu, ACS Appl. Mater. Interfaces, 2020, 12, 7631-7638.

2 T. Delipinar, A. Sha, M. S. Gohar and M. K. Yapici, ACS Omega, 2021, 6, 8744-8753.

3 A. Patil, R. Humbare and V. Kumar, Humidity Sensor Market by Product (Relative and Absolute) Type (Digital and Analog), and End Use (Residential, Commercial, Automotive, Industrial, Agriculture, Weather Station, and Healthcare): Global Opportunity Analysis and Industry Forecast, 2020-2027, 2020.

4 H. Farahani, R. Wagiran and M. N. Hamidon, Sensors, 2014, 14, 7881-7939.

5 J. Boudaden, M. Steinmaßl, H. Endres, A. Drost, I. Eisele, C. Kutter and P. Müller-buschbaum, Sensors, 2018, 18, 1516.

6 M. A. Najeeb, Z. Ahmad and R. A. Shakoor, Adv. Mater. Interfaces, 2018, 5, 1-19.

7 A. S. Afify, S. Ahmad, R. A. Khushnood, P. Jagdale and J. M. Tulliani, Sens. Actuators, B, 2017, 239, 1251-1256.

8 Z. Weng, J. Qin, A. A. Umar, J. Wang, X. Zhang, H. Wang, X. Cui, X. Li, L. Zheng and Y. Zhan, Adv. Funct. Mater., 2019, 29, 1-9.

9 D. Zhang, Z. Xu, Z. Yang and X. Song, Nano Energy, 2020, 67, 104251.

10 R. N. Jenjeti, R. Kumar and S. Sampath, J. Mater. Chem. A, 2019, 7, 14545-14551.

11 T. Kasuga, H. Yagyu, K. Uetani, H. Koga and M. Nogi, ACS Appl. Mater. Interfaces, 2019, 11, 43488-43493.

12 Z. Li, J. Wang, Y. Xu, M. Shen, C. Duan, L. Dai and Y. Ni, Carbohydr. Polym., 2021, 270, 118385.

13 Y. H. Jung, T. Chang, H. Zhang, C. Yao, Q. Zheng, V. W. Yang, H. Mi, M. Kim, S. J. Cho, D. Park, H. Jiang, J. Lee, Y. Qiu, W. Zhou, Z. Cai, S. Gong and Z. Ma, Nat. Commun., 2015, 6, 7170. 
14 B. De Morais, M. Santhiago, P. G. Costa, M. P. Pereira and C. C. B. Bufon, ACS Appl. Mater. Interfaces, 2018, 10, 35631-35638.

15 H. Liu, H. Xiang, Y. Wang, Z. Li, L. Qian, P. Li, H. Zhou and W. Huang, ACS Appl. Mater. Interfaces, 2019, 11, 40613-40619.

16 L. Dai, Y. Wang, X. Zou, Z. Chen, H. Liu and Y. Ni, Small, 2020, 16, 1-25.

17 Y. Wang, S. Hou, T. Li, S. Jin, Y. Shao, H. Yang, D. Wu, S. Dai, Y. Lu, S. Chen and J. Huang, ACS Appl. Mater. Interfaces, 2020, 12, 41896-41904.

18 F. Güder, A. Ainla, J. Redston, B. Mosadegh, A. Glavan, T. J. Martin and G. M. Whitesides, Angew. Chem., Int. Ed., 2016, 55, 5727-5732.

19 H. Tai, Z. Duan, Y. Wang, S. Wang and Y. Jiang, ACS Appl. Mater. Interfaces, 2020, 12, 31037-31053.

20 Z. Duan, Y. Jiang, M. Yan, S. Wang, Z. Yuan, Q. Zhao, P. Sun, G. Xie, X. Du and H. Tai, ACS Appl. Mater. Interfaces, 2019, 11, 21840-21849.

21 Z. Duan, Y. Jiang and H. Tai, J. Mater. Chem. C, 2021, 9, 14963-14980.

22 H. Zhao, T. Zhang, R. Qi, J. Dai, S. Liu and T. Fei, ACS Appl. Mater. Interfaces, 2017, 9, 28002-28009.

23 Z. Duan, Y. Jiang, Q. Zhao, Q. Huang, S. Wang, Y. Zhang, Y. Wu, B. Liu, Y. Zhen and H. Tai, Sens. Actuators, B, 2021, 339, 129884.

24 Z. Duan, Y. Jiang, Q. Huang, S. Wang, Y. Wang, H. Pan, Q. Zhao, G. Xie, X. Du and H. Tai, Smart Mater. Struct., 2021, 30, 055012.

25 T. Saito, Y. Nishiyama, J. L. Putaux, M. Vignon and A. Isogai, Biomacromolecules, 2006, 7, 1687-1691.
26 Y. Okita, T. Saito and A. Isogai, Biomacromolecules, 2010, 11, 1696-1700.

27 K. Lichtenstein and N. Lavoine, Polym. Degrad. Stab., 2017, 146, 53-60.

28 J. Kiefer, A. Stärk, A. L. Kiefer and H. Glade, Energies, 2018, 11, 798.

29 M. A. Pimenta, G. Dresselhaus, M. S. Dresselhaus, L. G. Cançado, A. Jorio and R. Saito, Phys. Chem. Chem. Phys., 2007, 9, 1276-1291.

30 J. Lin, Z. Peng, Y. Liu, F. Ruiz-Zepeda, R. Ye, E. L. G. Samuel, M. J. Yacaman, B. I. Yakobson and J. M. Tour, Nat. Commun., 2014, 5, 5-12.

31 N. Kurra, Q. Jiang, P. Nayak and H. N. Alshareef, Nano Today, 2019, 24, 81-102.

32 S. Lee and S. Jeon, ACS Sustainable Chem. Eng., 2019, 7, 2270-2275.

33 X. Guo, Y. Wu and X. Xie, Sci. Rep., 2017, 7, 14207.

34 H. Tai, S. Wang, Z. Duan and Y. Jiang, Sens. Actuators, B, 2020, 318, 128104.

35 M. Parrilla, T. Guinovart, J. Ferré, P. Blondeau, F. J. Andrade, M. Parrilla, T. Guinovart, J. Ferré, P. Blondeau and F. J. Andrade, Adv. Healthcare Mater., 2019, 8, 1900342.

36 Y. Lu, K. Xu, L. Zhang, M. Deguchi, H. Shishido, T. Arie, R. Pan, A. Hayashi, L. Shen, S. Akita and K. Takei, ACS Nano, 2020, 14, 10966-10975.

37 H. Skaar and A. Johnsson, Physiol. Plant., 1978, 43, 390-396.

38 A. De Luca, S. Santra, R. Ghosh, S. Z. Ali, J. W. Gardner, P. K. Guha and F. Udrea, Nanoscale, 2016, 8, 4565-4572.

39 M. Tannarana, P. M. Pataniya, S. A. Bhakhar, G. K. Solanki, J. Valand, S. Narayan, K. D. Patel, P. K. Jha and V. M. Pathak, ACS Sustainable Chem. Eng., 2020, 8, 12595-12602. 